\title{
Zeichen und Abkürzungen
}

\section{Zeichen}

[®] Textlücke; mit Text in lateinischen Buchstaben phonetische Angabe

\{N\} versehentliche Hinzufügung

$\langle\aleph \quad \quad$ versehentliche Auslassung

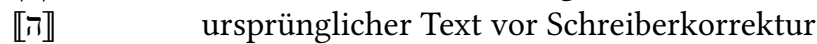

$\ll \aleph \gg \quad$ im Manuskript von einem Schreiber in $\boldsymbol{N}$ korrigiert

$+\ll \aleph \gg+\quad$ im Manuskript von einem Schreiber nachträglich eingefügt

- $\quad$ nicht deutbarer Zeichenrest

* im Manuskript getilgter Text

$\ulcorner\boldsymbol{K}\urcorner \quad$ Lesung am Rand

אוב $\quad$ Variante, die von einem Schreiber über einem Zeichen notiert wurde

Konjektur

// phonematische Angabe einer Grundform

> geworden zu

$<\quad$ geworden aus

$=\quad$ gleich

$\neq \quad$ ungleich

$\downarrow \quad$ Verweis auf aramäisches Lemma

$\rightarrow \quad$ Verweis auf hebräisches Lemma

\section{Allgemeine Abkürzungen}

$\begin{array}{llll}\text { ee } & \text { Samaritanus } & \text { äth. } & \text { äthiopische Sprachen } \\ \sigma^{\prime} & \text { Symmachus } & \text { af. } & \begin{array}{l}\text { Af'el } \\ \theta^{\prime}\end{array} \\ \gamma^{\prime} & \text { Theodotion } & \text { aff. } & \text { affirmativ } \\ \| & \text { die „Drei“ } & \text { akk. } & \text { akkadisch } \\ & (\mathrm{im}) \text { Parallelismus membrorum } & \text { allg. } & \text { allgemein } \\ & (\mathrm{zu}) & \text { amur. } & \text { amurritisch } \\ \text { a. } & \text { auch } & \text { Anspiel. } & \text { Anspielung } \\ \text { aaram. } & \text { altaramäisch } & \text { arab. } & \text { arabisch } \\ \text { Abl. } & \text { Ableitung(en) } & \text { aram. } & \text { aramäisch } \\ \text { abs. } & \text { Status absolutus } & \text { aramLW } & \text { aramäisches Lehnwort } \\ \text { Abstr. } & \text { Abstraktum } & \text { architek. } & \text { architektonisch } \\ \text { adj. } & \text { adjektivisch } & \text { a.Rd. } & \text { am Rand } \\ \text { Adj. } & \text { Adjektiv } & \text { asarab. } & \text { altsüdarabisch } \\ \text { adv. } & \text { adverbial } & \text { assyr. } & \text { assyrisch } \\ \text { Adv. } & \text { Adverb } & \text { attribut. } & \text { attributiv(isch) } \\ \text { äg. } & \text { ägyptisch } & \text { Aufzähl. } & \text { (in) Aufzählung } \\ \text { ähnl. } & \text { ähnlich } & \text { Ausl. } & \text { Auslegung }\end{array}$




\begin{tabular}{|c|c|c|c|}
\hline baram. & biblisch-aramäisch & ff. & folgende \\
\hline Bed. & Bedeutung & folg. & folgend $(\mathrm{em} / \mathrm{r})$ \\
\hline berb. & berberisch & frg. & Fragment \\
\hline bes. & besonders, besondere & $\mathrm{G}$ & Grundstamm \\
\hline Beschreib. & Beschreibung & $\mathfrak{G}$ & Septuaginta \\
\hline Bez. & Bezeichnung & $\mathfrak{G}^{\mathrm{B}}$ & Septuaginta (Vaticanus) \\
\hline bhe. & biblisch-hebräisch & $\mathfrak{G}^{\mathrm{S}}$ & Septuaginta (Sinaiticus) \\
\hline bibl. & biblisch & gaon. & gaonäisch \\
\hline bildl. & bildlich & gemeinsem. & gemeinsemitisch \\
\hline bzw. & beziehungsweise & gg. & gegen \\
\hline cj. & konjiziert & Ggs. & Gegensatz/Gegensätze \\
\hline comm. & communis & GN & Göttername \\
\hline $\begin{array}{l}\text { conj. } \\
\text { cparam. }\end{array}$ & $\begin{array}{l}\text { Konjunktion } \\
\text { christlich-palästinisch- } \\
\text { aramäisch }\end{array}$ & $\begin{array}{l}\text { Gottesbez. } \\
\text { göttl. } \\
\text { gramm. }\end{array}$ & $\begin{array}{l}\text { Gottesbezeichnung } \\
\text { göttlich } \\
\text { grammatikalisch }\end{array}$ \\
\hline cs. & Status constructus & griech. & griechisch \\
\hline $\mathrm{CsVb}$. & Constructus-Verbindung & Grundbed. & Grundbedeutung \\
\hline d. & der & haf. & Haf $^{\tau}$ el \\
\hline $\mathrm{D}$ & Doppelungsstamm & halakh. & halakhisch \\
\hline Dam. & Damaskusschrift & hap.leg. & Hapax legomenon \\
\hline def. & defektiv & he. & hebräisch \\
\hline deikt. & deiktisch & Hekh. & Hekhalot-Literatur \\
\hline denom. & denominiert & Hier. & Hieronymus \\
\hline det. & Status determinatus/determiniert & hif. & Hif'il \\
\hline dgg. & dagegen & himml. & himmlisch \\
\hline d.h. & das heißt & hist. & historisch \\
\hline dial. & dialektal & hitp. & Hitpa ${ }^{c} e l$ \\
\hline Dittogr. & Dittographie & hitpal. & Hitpalpel \\
\hline du. & dual(isch) & hitpo. & Hitpolel \\
\hline ebd. & ebenda & Hod. & Hodajot \\
\hline ebenf. & ebenfalls & hof. & Hof ${ }^{\mathrm{t}} \mathrm{al}$ \\
\hline ebla. & eblaitisch & hurr. & hurritisch \\
\hline eig. & eigentlich & hymn. & hymnisch \\
\hline emph. & emphatisch & i. & $\mathrm{in} / \mathrm{m}$ \\
\hline endzeitl. & endzeitlich & i.A.d. & in Aufnahme der \\
\hline energ. & Energicus & i.A.v. & in Aufnahme von \\
\hline entspr. & entsprechend & i.Bz.a. & in Bezug auf \\
\hline Epiph. & Epiphanius & id. & idumäisch-aramäisch \\
\hline erg. & ergänzt & i.d.B. & in der Bedeutung \\
\hline Erg. & Ergänzung & i.Ggs.z. & im Gegensatz zu \\
\hline eschatol. & eschatologisch & i.K. & im Kontext \\
\hline et al. & und andere & imp. & Imperativ \\
\hline etym. & etymologisch & impf. & Imperfekt \\
\hline Etym. & Etymologie & impf.cons. & Imperfekt consecutivum \\
\hline Eus. & Euseb & inf. & Infinitiv \\
\hline f. & Femininum & insbes. & insbesondere \\
\hline Fasc. & Fascicle & inschr. & inschriftlich \\
\hline
\end{tabular}




\begin{tabular}{|c|c|c|c|}
\hline interj. & Interjektion & mhe. $2 b$ & $\begin{array}{l}\text { mittelhebräisch, amoräisch, aus } \\
\text { Babylonien }\end{array}$ \\
\hline intrans. & intransitiv & & Babylonien \\
\hline i.S.v. & im Sinne von & mhe. $2 p$ & mittelhebräisch, amoräisch, aus \\
\hline itpa. & Itpacal & & Palästina \\
\hline i.u.K. & in unsicherem Kontext & mhe.b & mittelhebräisch, aus Babyloni- \\
\hline i.Zshg. & im Zusammenhang & & en \\
\hline jaram. & jüdisch-aramäisch & mhe.p & mittelhebräisch, aus Palästina \\
\hline jbaram. & jüdisch-babylonisch-aramäisch & MidrEschat & Midrasch zur Eschatologie \\
\hline Jh. & Jahrhundert & Milh. & Kriegsregel \\
\hline jmd. & jemand(en/em) & milit. & militärisch \\
\hline Flav.Jos. & Flavius Josephus & MLM & Musar le-Mevin (Instructions) \\
\hline param. & jüdisch-palästinisch-aramäisch & MMT & Miqșat Ma'aśe ha-Tora \\
\hline jtaram. & jüdisch-targumisch-aramäsich & M & ame \\
\hline juss. & Jussiv & moab. & mo \\
\hline K. & Kontext & mögl. & erweise) \\
\hline kalend. & kalendarisch & mpers. & mittelpersisch \\
\hline kan. & kanaanäisch & M & Mar \\
\hline keilschr. & keilschriftlich & Mss. & ripte \\
\hline $\mathrm{KF}$ & Kurzform & Myst. & My \\
\hline königl. & königlich & $\mathrm{n}$. & Nor \\
\hline toh. & Kohortativ & nassyr. & syrisch \\
\hline koll. & kollektiv & neg. & neg \\
\hline Konj. & Konjunktion & Neg. & Negation \\
\hline korr. & korrigiert & NF & Nebenform \\
\hline kosmol. & kosmologisch & nichtsem. & nichtsemitisch \\
\hline xult. & kultisch & nif. & $\mathrm{Nif}^{\mathrm{r} a l}$ \\
\hline Kurzimpf. & Kurzimperfekt & nitpa. & Nitpa'el \\
\hline 1. & lies $(\mathrm{t})$, lesen & $\mathrm{n}$. & Nomen \\
\hline L. & Lesung & n.c. & imunis \\
\hline Langimpf. & Langimperf & n.d. & Göttername \\
\hline lat. & $\begin{array}{l}\text { Langimperi } \\
\text { lateinisch }\end{array}$ & neg. & $\mathrm{Ne}$ \\
\hline liturg. & $\begin{array}{l}\text { late1 } \\
\text { litur }\end{array}$ & n.f. & femininum \\
\hline loc. & $\begin{array}{l}\text { liturgisch } \\
\text { locativ }\end{array}$ & n.fl. & Flußname \\
\hline L.u. & $\begin{array}{l}\text { loca } \\
\text { Lesu }\end{array}$ & n.l. & Ort \\
\hline LW & $\begin{array}{l}\text { Lesur } \\
\text { Lehn }\end{array}$ & n.m. & Nomen maskulinum \\
\hline & & not.acc. & Objektmarker (nota accusativi) \\
\hline m. & Mask & n.p. & Volksname \\
\hline $\mathfrak{M}$ & masoretischer Text & n.pr. & Personenname \\
\hline ma. & mittelalterlich & nsarab. & neusüdarabisch \\
\hline mahe. & alterlich-hebräisch & n.t. & Gebietsname \\
\hline mand. & mandäisch & n.top. & Landschaftsname \\
\hline mas. & masoretisch & n.un. & Nomen unitatis \\
\hline menschl. & menschlich & n.u.Z. & nach unserer Zeitrechnung \\
\hline meton. & misch & nwsem. & nordwestsemitisch \\
\hline mhe. & ittelhebräisch & o.ä. & oder ähnlich \\
\hline mhe. 1 & nebräisch, tannaitisch & o. $\ddot{A}$. & oder Ähnliches \\
\hline nhe.2 & mittelhebräisch, amoräisch & oaram. & ostaramäisch \\
\hline
\end{tabular}




\begin{tabular}{|c|c|c|c|}
\hline Obj. & Objekt & $\mathrm{pu}^{\mathrm{c} a l}$ & Pual \\
\hline od. & oder & pulal & $\mathrm{Pu}$ lal \\
\hline o.O. & ohne Ort & pun. & punisch \\
\hline orth. & orthographisch & qal & Qal \\
\hline P. & Pescher & qal $_{\text {pass. }}$ & Qal passiv \\
\hline $\mathrm{P}$ & Priesterschrift/priesterschriftlich & qaram. & qumran-aramäisch \\
\hline pa. & $\mathrm{Pa}^{\mathrm{cc}} \mathrm{el}$ & $\mathrm{qb}$. & qumran-biblisch \\
\hline pal. & palästinisch & qhe. & qumran-hebräisch \\
\hline paläogr. & paläographisch & raram. & reichsramäisch \\
\hline palm. & palmyrenisch & räuml. & räumlich \\
\hline par. & Parallelhandschrift(en) & relig. & religiös \\
\hline paragog. & paragogicum & rhet. & rhetorisch \\
\hline param. & palästinisch-aramäisch & Rndb. & Randbemerkung \\
\hline part.adv. & adverbiale Partikel & $\mathrm{S}$ & Serekh ha-Jachad \\
\hline part.interr. & Fragepartikel & $\mathfrak{S}$ & Peschitta \\
\hline part.rel. & Relativpartikel & s. & siehe \\
\hline praep. & Präposition & s.a. & siehe auch \\
\hline pass. & passiv & sab. & sabäisch \\
\hline paus. & pausal & samar. & samaritanisch \\
\hline pe. & $\mathrm{Pe}^{c} \mathrm{al}$ & saram. & samaritanisch-aramäisch \\
\hline pers. & persisch & Schreibf. & Schreibfehler \\
\hline pf. & Perfekt & scil. & scilicet \\
\hline pf.cons. & Perfekt consecutivum & s.d. & siehe dort \\
\hline $\mathrm{pHab}$ & Pescher Habakuk & Sec. & Secunda \\
\hline phön. & phönizisch & sek. & sekundär \\
\hline physiogn. & physiognomisch & sem. & semitisch \\
\hline pi. & $\mathrm{Pi}^{\mathrm{c}} \mathrm{el}$ & seman. & sematisch \\
\hline pil. & Pilpel & sf. & Suffix \\
\hline piyyut. & $\begin{array}{l}\text { (Hebräisch der) Piyyutim und } \\
\text { verwandte(r) Literatur }\end{array}$ & $\begin{array}{l}\text { sg. } \\
\text { she. }\end{array}$ & $\begin{array}{l}\text { Singular, singularisch } \\
\text { samaritanisch-hebräisch }\end{array}$ \\
\hline PK & Präformativkonjugation & ShirShabb & Sabbatlieder \\
\hline pl. & Plural, pluralisch & Sir & Sirach \\
\hline po. & Polel & $\operatorname{Sir}^{\mathrm{A}}$ & Sirach, Manuskript A \\
\hline poet. & poetisch & $\operatorname{Sir}^{B}$ & Sirach, Manuskript B \\
\hline pos. & positiv & $\mathrm{Sir}^{\mathrm{C}}$ & Sirach, Manuskript C \\
\hline präd. & prädikativ & $\operatorname{Sir}^{\mathrm{D}}$ & Sirach, Manuskript D \\
\hline Präd. & Prädikat & $\operatorname{Sir}^{\mathrm{E}}$ & Sirach, Manuskript E \\
\hline präp. & präpositional & $\operatorname{Sir}^{\mathrm{F}}$ & Sirach, Manuskript F \\
\hline $\begin{array}{l}\text { Präp. } \\
\text { priesterl. }\end{array}$ & $\begin{array}{l}\text { Präposition } \\
\text { priesterlich }\end{array}$ & Sirma & $\begin{array}{l}\text { Sirach, mittelalterliche Manu- } \\
\text { skripte }\end{array}$ \\
\hline $\mathrm{PrN}$ & Primärnomen & SirMas & Sirach, Manuskript aus Masada \\
\hline prohib. & Prohibitiv & s.o. & siehe oben \\
\hline pron. & Pronomen & sog. & sogenannt \\
\hline pron.dem. & Demonstrativpronomen & soz. & sozial \\
\hline pron.interr. & Fragepronomen & sp. & spät(es) \\
\hline prosth. & prostheticum & spbab. & spätbabylonisch \\
\hline ptz. & Partizip & spmahe. & spätmittelalterlich-hebräisch \\
\hline
\end{tabular}




\begin{tabular}{|c|c|c|c|}
\hline sprachl. & sprachlich & ursem. & ursemitisch \\
\hline st.rect. & Status rectum & urspr. & ursprünglich \\
\hline st.reg. & Status regens & v. & von \\
\hline s.u. & siehe unten & $\mathrm{V}$. & Vers \\
\hline Subj. & Subjekt & $\mathfrak{V}$ & Vulgata \\
\hline subst. & substantivisch & Var. & Variante \\
\hline sum. & sumerisch & vb. & Verb(wurzel), verbal \\
\hline s.v. & sub voce & $\mathrm{Vb}$. & Verbindung \\
\hline syr. & syrisch & verallg. & verallgemeinernd \\
\hline $\mathfrak{T}$ & Targum & vereinz. & vereinzelt \\
\hline Tele & Samaritanisches Targum & vermutl. & vermutlich \\
\hline TeuC & $\begin{array}{l}\text { Samaritanisches Targum, Ma- } \\
\text { nuskript C }\end{array}$ & verschied. & verschieden $(\mathrm{er} / \mathrm{s})$ \\
\hline $\mathfrak{T J}$ & Targum Jonatan & & verwandt \\
\hline $\mathfrak{T}^{\mathrm{N}}$ & Targum Neofiti & vgl. & ver \\
\hline$\tilde{\mathfrak{T}}^{\mathrm{O}}$ & Targum Onkelos & Vgl. & Vergleich \\
\hline$\tilde{\mathfrak{T}} \mathrm{PsJ}$ & Targum Pseudo-Jonatan & viell. & vielleicht \\
\hline eilv & teilweise & Vok. & Vokalisierung \\
\hline emp. & temporal & vok.b & babylonisch vokalisiert \\
\hline & targumisch & vok.p & palästinisch vokalisiert \\
\hline $\operatorname{tg} \cdot b$ & babylonisch-targumisch & Vol. & Volume \\
\hline TR & Tempelrolle & vorausgeh. & vorausgehend \\
\hline rad. & traditionell & vorgeschl. & vorg \\
\hline Trad. & Tradition & wahrsch. & wahrscheinlich \\
\hline rans. & transitiv & waram. & westaramäisch \\
\hline t.t. & Terminus technicus & weisheitl. & $\begin{array}{l}\text { weisheitlich } \\
\text { wörtlich }\end{array}$ \\
\hline u. & und & wsem. & westsemitisch \\
\hline $\begin{array}{l}\text { u.a. } \\
\text { u.ä. }\end{array}$ & $\begin{array}{l}\text { und andere(s) } \\
\text { und ähnlich }\end{array}$ & Wz. & Wurzel \\
\hline u. $\ddot{\text { A. }}$ & und Ähnliches & $\mathrm{Z}$. & Zeile \\
\hline Übers. & Übersetzung & z.B. & zum Beispiel \\
\hline übertr. & übertragen & zeitl. & zeitlich \\
\hline ug. & ugaritisch & Zit. & Zitat \\
\hline ג.ö. & und öfter & Zshg. & Zusammenhang \\
\hline insich. & unsicher & z.St. & zur Stelle \\
\hline nwahrsc & unwahrscheinlich & $\mathrm{zW}$. & zwischen \\
\hline
\end{tabular}

\section{Bibel}

Gen Ex Lev Num Dtn Jos Ri 1 Sam 2 Sam 1 Kön 2 Kön Jes Jer $\mathrm{Ez}$ Hos Joel Amos $\mathrm{Ob}$ Jona $\mathrm{Mi} \mathrm{Nah}$ Hab Zeph Hag Sach Mal Ps Hi Prov Rut Hhld Qoh Klgl Est Dan Esr Neh 1 Chron 2 Chron
Röm
Römerbrief
Apk
Apokalypse
2 Tim
2 Timotheus 


\section{Apokryphen und Pseudepigraphen}

$\begin{array}{ll}1 \text { Hen } & 1 \text { Henoch } \\ 1 \text { Makk } & 1 \text { Makkabäer } \\ \text { Jub } & \text { Jubiläen }\end{array}$

TestNaph Testament Naphtalis

Tob Tobit

\section{Rabbinische Quellen}

Mischna (m...), Tosefta (t...) und Talmud (b... = babylonischer, $\mathrm{j} \ldots=$ Jerusalemer Talmud)

$\begin{array}{llll}\text { Av } & \text { Avot } & \text { Mak } & \text { Makkot } \\ \text { AZ } & \text { Avoda Zara } & \text { Meg } & \text { Megilla } \\ \text { BB } & \text { Bava Batra } & \text { Meil } & \text { Me'ila } \\ \text { Bekh } & \text { Bekhorot } & \text { Mid } & \text { Middot } \\ \text { Ber } & \text { Berakhot } & \text { Men } & \text { Menachot } \\ \text { Bez } & \text { Beza } & \text { MQ } & \text { Mo'ed Qatan } \\ \text { BM } & \text { Bava Mezi'a } & \text { Ned } & \text { Nedarim } \\ \text { BQ } & \text { Bava Qamma } & \text { Oh } & \text { Ohalot } \\ \text { Chag } & \text { Chagiga } & \text { Pes } & \text { Pesachim } \\ \text { Chul } & \text { Chullin } & \text { San } & \text { Sanhedrin } \\ \text { Er } & \text { Eruvin } & \text { Shab } & \text { Shabbat } \\ \text { Git } & \text { Gittin } & \text { Sheq } & \text { Sheqalim } \\ \text { Jev } & \text { Jevamot } & \text { Shevi } & \text { Shevi'it } \\ \text { Kel } & \text { Kelim } & \text { Shevu } & \text { Shevu'ot } \\ \text { Ker } & \text { Keritot } & \text { Suk } & \text { Sukka } \\ \text { Ket } & \text { Ketubbot } & \text { Taan } & \text { Ta'anit } \\ \text { Kil } & \text { Kil’ajim } & \text { Zev } & \text { Zevachim } \\ \text { Maas } & \text { Ma'asrot } & \end{array}$

Midraschim

$\begin{array}{llll}\text { ARN B } & \text { Avoth de Rabbi Natan B } & \text { MekhSh } & \begin{array}{l}\text { Mekhilta deRabbi Shim on bar } \\ \text { BemR }\end{array} \\ \text { Bemidbar Rabba } & \text { MidrPss } & \text { Midrasch Psalmen } \\ \text { BerR } & \text { Bereshit Rabba } & \text { PRE } & \text { Pirqe deRabbi Eli'eser } \\ \text { BerRbti } & \text { Bereshit Rabbati } & \text { QohR } & \text { Qohelet Rabba } \\ \text { DER } & \text { Derekh Erez Rabba } & \text { Sem } & \text { Semachot } \\ \text { DES } & \text { Derekh Erez Suta } & \text { SER } & \text { Seder Elijahu Rabba } \\ \text { DevR } & \text { Devarim Rabba } & \text { ShemR } & \text { Shemot Rabba } \\ \text { EkhR } & \text { Ekha Rabba(ti) } & \text { ShirR } & \text { Shir HaShirim Rabba } \\ \text { HekhRab } & \text { Hekhalot Rabbati } & \text { SifBem } & \text { Sifre Bemidbar } \\ \text { JalqShim } & \text { Jalqut Schim`oni } & \text { SifDev } & \text { Sifre Devarim } \\ \text { KallaR } & \text { Kalla Rabbati } & \text { SifS } & \text { Sifre Suta } \\ \text { MekhJ } & \text { Mekhilta deRabbi Jishmacel } & \text { Tan } & \text { Tanhuma }\end{array}$




\section{Andere Quellen}

B-.. $\quad$ Bildnummer der Leon Levy Dead Sea Scrolls Digital Library, http://www.deadseascrolls.org.il/

EA 245,28 Text aus El Amarna

EusOn Euseb, Onomastikon

FGP No.... Objektnummer des Friedberg Genizah Project, http://www.jewishmanuscript.org/

Flav.Jos.Ant. Flavius Josephus, Antiquitates

Frah. H.S. Nyberg, Frahang I Pahlavîk. Edited with Transliteration, Transcription and Commentary from the Posthumous Papers of Henrik Samuel Nyberg by Bo Utas with the Collaboration of Christopher Toll, Wiesbaden 1988

Id.Ostr. B. Porten, Textbook of Aramaic Ostraca from Idumea 1-2, Winona Lake, Indiana, 2014-16

KAI H. Donner/W. Röllig, Kanaanäische und aramäische Inschriften 1, Wiesbaden ${ }^{5} 2002$

KTU M. Dietrich/O. Loretz/J. Sanmartin, Die keilalphabetischen Texte aus Ugarit, Ras Ibn Hani und anderen Orten (Alter Orient und Altes Testament 360/1), Münster 2013

Lak J. Renz, Die althebräischen Inschriften 1. Text und Kommentar. Handbuch der althebräischen Epigraphik 1, Darmstadt 1995

pTurin Papyrus Turin

Qas J. Renz, Die althebräischen Inschriften 1. Text und Kommentar. Handbuch der althebräischen Epigraphik 1, Darmstadt 1995

TAD B. Porten/A. Yardeni, Textbook of Aramaic Documents from Ancient Egypt. 14, Jerusalem 1986-99

T-S Taylor/Schechter 
